Cahiers de recherches médiévales

\title{
La nourriture et le corps au Moyen Âge
}

Danielle Jacquart

\section{(2) OpenEdition}

Journals

Édition électronique

URL : https://journals.openedition.org/crm/868

DOI : $10.4000 / \mathrm{crm} .868$

ISSN : 1955-2424

Éditeur

Honoré Champion

Édition imprimée

Date de publication : 30 mars 2006

Pagination : 259-266

ISSN : $1272-9752$

Référence électronique

Danielle Jacquart, «La nourriture et le corps au Moyen Âge », Cahiers de recherches médiévales [En ligne], 13 spécial | 2006, mis en ligne le 03 avril 2009, consulté le 15 décembre 2022. URL : http:// journals.openedition.org/crm/868; DOI : https://doi.org/10.4000/crm.868 


\title{
RM
}

\section{La nourriture et le corps au Moyen Âge}

Les folios d'ouverture d'un manuscrit italien du XIV siècle, transcrivant l'ouvrage de botanique médicale dû à Manfred de Monte Imperiali, donnent à voir, en une savoureuse illustration, un échange de propos entre savants du passé. C'est ainsi que de la bouche de Galien sort, adressée à Hippocrate, la célèbre sentence, attribuée depuis l'Antiquité à divers auteurs et reprise par Molière dans L'Avare: Intendo enim manducare ut vivam, alii intendunt vivere ut manducent ( $\mathrm{J}$ 'entends manger pour vivre, d'autres entendent vivre pour manger »). Le médecin rejoint ici le moraliste en tenant à l'écart la gloutonnerie, tout en reconnaissant évidemment la nécessité vitale de la nutrition. L'illustration du manuscrit italien prête encore un autre propos à Galien, qui cette fois ne sort pas directement de sa bouche, mais, un peu plus technique, est écrit sur le rouleau qu'il tient à la main : Quicumque vult continuam custodire sanitatem, custodiat stomachum ne cum sibi necessarium sit prohibeat cibum («Qui veut garder toujours la santé doit préserver son estomac pour qu'il n'empêche pas la nutrition alors qu'elle lui est nécessaire»). Avec cette phrase s'introduit la diététique, indispensable au bon fonctionnement de l'estomac, et par voie de conséquence garante de la santé.

\section{De la médecine à la cuisine et à la diététique}

Les prescriptions alimentaires sont présentes dès les plus anciens ouvrages de médecine parvenus jusqu'à nous. À la fin $d u \mathrm{~V}^{\mathrm{e}}$ siècle avant Jésus Christ, l'auteur du traité hippocratique Ancienne médecine se livrait à une réflexion d'ordre anthropologique, en faisant de l'invention de la cuisine, puis de la médecine, deux étapes fondamentales dans la genèse de l'homme civilisé. Au tout début, dit l'auteur hippocratique, les deux arts étaient confondus; il choisit de les réunir sous l'appellation commune de «médecine» du fait que le but visé était la santé. Après avoir constaté qu'ils ne pouvaient sans dommage se nourrir comme les animaux, les hommes passèrent non seulement au cuit, mais à des préparations élaborées :

\begin{abstract}
Ainsi donc, à partir des grains de blé, après les avoir mouillés, mondés, moulus, tamisés, pétris et cuits, ils confectionnèrent le pain, et à partir des grains d'orge, la galette. Et procédant à bien d'autres opérations pour préparer cette nourriture, ils firent bouillir et rôtir, mêlèrent et tempérèrent les substances fortes et intempérées à l'aide de substances plus faibles, façonnant tout en conformité avec la capacité naturelle de l'homme; car ils estimaient que, dans le cas des aliments trop forts, la nature de l'homme ne sera pas capable de les dominer si elle les ingère, et qu'il en résultera de ces aliments eux-mêmes souffrances, maladies et mort, tandis que de tous les aliments qu'elle est capable de dominer, il résultera nourriture, accroissement et santé.
\end{abstract}

${ }^{1}$ Paris, Bibliothèque nationale de France, manuscrit latin 6823 , fol. $1^{\mathrm{v}}$, reproduit dans : M. J. Imbault-Huart, La médecine au Moyen Âge à travers les manuscrits de la Bibliothèque Nationale, Paris, 1983, p. 41.

Cahiers de Recherches Médiévales, 13spé, 2006 
Or, à cette découverte et à cette enquête, quel nom plus juste ou plus adéquat pourrait-on donner que celui de médecine, puisqu'il s'agit d'une découverte faite pour la santé, le salut et la nourriture de l'homme, en remplacement de ce régime-là qui était à l'origine des souffrances, des maladies et de la mort ?²

Dans une étape ultérieure de la civilisation, il fut découvert qu'un même régime ne pouvait convenir aux malades et aux bien portants. Intervint alors «la médecine reconnue comme telle». On commença par réduire la quantité absorbée, mais, comme cela ne s'avéra pas suffisant pour certains malades, une gradation fut peu à peu établie :

[...] ils découvrirent les potages, en mélangeant une petite quantité de substances fortes à beaucoup d'eau et en ôtant la force de ces substances par le mélange et la cuisson. Enfin, pour les malades qui ne pouvaient même pas triompher des potages, ils retranchèrent aussi ces potages et en vinrent aux boissons; encore veillèrent-ils à ce qu'elles fussent dans une juste mesure tant par le mélange que par la quantité, s'abstenant d'administrer des boissons trop abondantes et trop intempérées ou aussi trop insuffisantes ${ }^{3}$.

Selon ce récit, cuisine et médecine sont donc des sortes de sœurs siamoises, qui se sont séparées au fur et à mesure des progrès de la civilisation. Réunies à l'origine pour offrir à l'ensemble de l'humanité une diététique, l'une d'entre elles s'est distinguée par l'attention portée à la nourriture spécifique des malades. Ce que ne dit pas explicitement ici l'auteur d'Ancienne Médecine, c'est que la médecine n'a pas pour autant laissé l'alimentation des bien portants à la seule cuisine. S'est créée en son sein une branche consacrée à la préservation de la santé, incluant des prescriptions alimentaires, dont d'autres textes hippocratiques, comme Nature de l'Homme ou le Régime sont les fondateurs.

Cuisine et médecine ont pour point commun l'art du mélange, imitant ainsi la nature qui elle aussi a œuvré et continue à œuvrer avec des mélanges. Dans la tradition hippocratique, prolongée par Galien et les auteurs arabes, et avec l'appui de la philosophie naturelle d'Aristote, la représentation médiévale du corps humain en fait le résultat d'une succession de mélanges, dont les justes proportions constituent l'état de santé. L'adage "manger pour vivre et non vivre pour manger» se traduit, en termes médiévaux, par la recherche d'un bon équilibre, grâce à la nourriture, des mélanges constitutifs du corps humain qui caractérisent sa ou ses complexion(s). À chaque partie du corps est attribuée une norme de complexion, par exemple le cerveau est froid et humide. Sous la tendance générale de la complexion humaine, chaude et humide, chaque individu répond à une norme, qu'il tient de son sexe, de son âge, de son appartenance régionale, mais aussi de sa nature propre, déterminée à sa naissance. Au-delà du maintien de cet équilibre, la nourriture restaure le corps. Elle reconstitue au sens propre du terme les substances dont il est formé, au fur et à mesure de la consomption de celles-ci sous l'effet des activités qu'accomplit le corps grâce à des facultés ou «vertus ». Les fonctions de digestion, d'assimilation et

\footnotetext{
${ }^{2}$ Ancienne Médecine, chap. 3, cf. J. Jouanna, Hippocrate, Paris, 1992, p. 331-332.

${ }^{3}$ Ancienne Médecine, chap. 5, ibid., p. 333.
} 
de croissance incombent à la faculté dite «naturelle», que contrairement à Galien, une majorité d'auteurs médiévaux, à partir du XIII ${ }^{\mathrm{e}}$ siècle, place sous le commandement de l'âme, comme les autres facultés corporelles ${ }^{4}$.

Si les nourritures ingérées peuvent reconstituer les substances du corps, c'est parce que les unes et les autres ont une matière similaire. À la différence des corps célestes incorruptibles, tous les êtres, animés ou inanimés, du monde sub-lunaire ont été façonnés, au moment de la Création, à partir des quatre éléments ${ }^{5}$. Cette matière primordiale est donc commune au corps de l'homme et aux produits qu'il ingère, qu'ils soient d'origine végétale, animale ou minérale. Pour façonner la substance humaine (ou animale en général), il fallut une étape supplémentaire d'élaboration, la transformation des éléments en humeurs, qui en sont les «filles", selon l'expression utilisée à la fin du XI ${ }^{\mathrm{e}}$ siècle par Constantin l'Africain, dans son Pantegni ${ }^{6}$. Chacune des quatre humeurs conserve les qualités de l'élément qui a dominé lors de son élaboration : le sang est homologue de l'air, car il est chaud et humide, les deux qualités caractéristiques de la vie; le phlegme, froid et humide est homologue de l'eau; la bile, chaude et sèche, est homologue du feu; enfin la mélancolie, froide et sèche, est homologue de la terre. Dotées de ces qualités, issues du mélange des éléments, les humeurs par leurs propres mélanges ont façonné toutes les parties solides et homogènes du corps humain: les os, la chair, les nerfs, les veines etc. L'union et l'agencement de ces parties homogènes ont constitué les parties dites composées ou instrumentales, à savoir les organes, tels que le cœur ou le foie, mais aussi des membres, tels que le bras ou la main. La différenciation entre les parties homogènes du corps vient d'une prédominance de l'une ou l'autre humeur dans le mélange qui les a constituées ; pour assurer leur régénération, il est nécessaire que l'alimentation intègre ces différences.

Le processus de fabrication de la matière corporelle, tel qu'il est imaginé à l'origine de l'homme, se reproduit par la génération dans la formation de l'embryon, sauf qu'il n'y a évidemment pas de retour aux quatre éléments primordiaux. Ce sont les quatre humeurs, représentées dans les semences parentales, qui vont par leurs mélanges fournir la matière des différentes parties du corps du fœtus. Ce sont elles, ou plutôt leurs sous-produits, qui assurent, tout au long de la vie, la reconstitution des substances corporelles. En dehors de toute notion de circulation, le sang qui s'écoule dans les veines et les artères sert, en effet, à irriguer et doit être constamment renouvelé à partir de la nutrition. Il n'est pas constitué de la seule humeur appelée «sang»,

\footnotetext{
${ }^{4} \mathrm{Au}$ début de son traité Des facultés naturelles, Galien distingue les facultés selon qu'elles relèvent ou non de l'âme : «Puisque la sensation et le mouvement volontaire sont propres aux animaux, tandis que la nutrition et l'accroissement sont communs aux animaux et aux plantes, ces opérations doivent être attribuées, les premières à l'âme, les secondes à la nature » (trad. de C. Daremberg, dans Galien, Euvres médicales choisies, éd. A. Pichot, Paris, 1994, p. 3). Pour Avicenne, l'âme commande aussi la nutrition, par l'intermédiaire de ses puissances végétatives, cf. Avicenna Latinus, Liber de anima seu Sextus de naturalibus, I-III, éd. de S. Van Riet, Louvain-Leyde, 1972, p. 79-82.

${ }^{5}$ Cette théorie repose sur les principes énoncés par Aristote dans De la génération et de la corruption : cf. éd. et trad. de C. Mugler, Paris, 1966 (Collection des Universités de France).

${ }^{6}$ Le Pantegni est une adaptation fort libre d'une encyclopédie médicale arabe, écrite à la fin du $\mathrm{X}^{\mathrm{e}}$ siècle par l'auteur persan al-Majûsî. Il a fait l'objet d'éditions à la Renaissance : Lyon 1515 et Bâle 1539 .
} 
mais de l'ensemble des quatre. Si des organes sont les réceptacles de certaines de ces humeurs, la vésicule biliaire pour la bile, la rate pour la mélancolie, ils n'exercent pas de fonction de sécrétion: ils ne servent qu'à contenir un surplus, aidant à la digestion ou à un rééquilibrage humoral. L'essentiel de la bile et de la mélancolie demeure dans la masse sanguine et pourvoit à la régénération de la substance corporelle.

\section{L'assimilation de l'aliment}

Dans son Canon de la médecine, traduit par Gérard de Crémone à Tolède dans la seconde moitié du XII ${ }^{\mathrm{e}}$ siècle, le philosophe et médecin persan Avicenne (mort en 1037) fournissait une description de la digestion et de la fabrication des humeurs, inspirée de Galien, qui servit de point d'appui principal à la représentation médiévale. Mettant à profit cette description, ainsi que d'autres lectures, Pietro d'Abano, dans l'une des 210 questions qui forment son vaste Conciliator, terminé à Padoue en 1310, revient avec précision sur l'élaboration des différentes matières corporelles. L'interrogation porte sur le caractère successif ou simultané de la fabrication des humeurs. Nous nous référerons à ces deux auteurs, Avicenne et Pietro d'Abano, pour suivre la transformation de l'aliment à travers le corps?

Livrons-nous d'abord à une récapitulation des différents types de substances dont est formé le corps humain. Sa structure anatomique lui est donnée par des parties solides qui, comme il a été dit, sont élaborées, au stade embryonnaire, à partir des humeurs et renouvelées par celles-ci au cours de la vie. Les fonctions du corps sont commandées par des facultés, émanant de l'âme, immatérielles, que transmettent des souffles, des pneumas ou « esprits » : la substance de ceux-ci, très subtile, est composée à la fois à partir de la vapeur issue de la cuisson des humeurs, sous l'effet de la chaleur naturelle, et de l'air apporté par la respiration. Enfin, le corps humain est composé de fluides, de substances liquides, au premier rang desquels se trouvent les quatre humeurs ${ }^{8}$.

Avicenne définit l'humeur comme un corps humide, liquide, dans lequel l'aliment est en premier lieu converti. Tout l'aliment n'est cependant pas converti en humeur : aux divers stades de la digestion, interviennent des surplus qui, soit servent à élaborer d'autres substances utiles, soit sont expulsés. Les quatre humeurs forment ce qu'Avicenne appelle «les humidités premières». Mais le corps abrite aussi des «humidités secondes», de quatre types. L'une d'entre elles, appelée «humide radical», est congénitale: d'origine spermatique, elle assure, en une sorte de colle, la

${ }^{7}$ Avicenne, Canon, livre I, fen 1, doctrine 4, chapitre 1. La version de Gérard de Crémone a été éditée à plusieurs reprises à la Renaissance, à partir de 1473. Je me réfère à l'édition de Lyon 1498 dans laquelle le texte d'Avicenne est accompagné du commentaire de Jacques Despars. Pietro d'Abano, Conciliator, éd. Venise 1565 (réimpr. Padoue, 1985), diff. XXIX. Sur la question de l'utilité nutritive des autres humeurs que le sang, et les débats qu'elle a suscités, voir D. Jacquart, La Médecine médiévale dans le cadre parisien, XIV $V^{e} X V^{e}$ siècle, Paris 1998, p. 333-347.

${ }^{8}$ Pour une présentation générale des cadres de la doctrine médicale, voir N. G. Siraisi, Medieval and Early Renaissance Medicine. An Introduction to Knowledge and Practice, Chicago, 1990. 
continuité des parties du corps qui lui donne sa caractéristique humaine. L'humide radical se consume tout au long de la vie : lorsqu'il est épuisé, la mort de l'individu intervient inéluctablement. Dans la stricte doctrine médicale, il ne peut être régénéré ni par l'alimentation, ni par une médication quelconque. Tout au plus, le médecin s'efforce-t-il d'éviter un assèchement pathologique qui abrègerait le terme naturel de la vie de son patient. La régénération de cet humide radical fut l'une des quêtes des alchimistes, à la recherche de moyens pour prolonger la viè .

À côté de cette humidité seconde, congénitale, Avicenne en énumère trois autres, produites aux différents stades de transformation des humeurs : constamment renouvelées, elles vont assurer la régénération des substances corporelles. Au premier stade, c'est l'humidité «sans nom» qui sort des plus petits vaisseaux irriguant le corps; au second stade, elle est appelée «rosée», car comme la rosée elle reste à la surface des parties anatomiques homogènes pour les humidifier et, au besoin, se transformer encore; au troisième stade, c'est l'humidité dite "change», car sous l'effet d'une coagulation, elle fournit la matière nécessaire à la régénération de la partie du corps où elle se trouve. La transformation ultime de ce «change» en substance corporelle relève, selon les mots d'Avicenne, du «divin». Cela ne signifie évidemment pas qu'il y faut une intervention divine, mais que l'ultime transformation est réglée par la partie du corps en attente de régénération, en fonction d'une vertu dont elle détient seule le secret. Cette propriété lui fait attirer et assimiler le «change» dont elle a besoin. Cette représentation médicale de la transformation de l'aliment en corps suscita quelque émoi chez des théologiens, soucieux de maintenir l'impossibilité d'une altération de la «vérité de la nature humaine ${ }^{10}$. En réalité, la doctrine médicale elle-même incluait ses propres garde-fous pour que des esprits audacieux ne puissent tenter de changer, par l'alimentation, le corps humain au-delà des limites naturelles voulues par Dieu. D'une part, l'impossibilité postulée d'une régénération de l'humide radical excluait les tentations d'immortalité et garantissait la pérennité, au niveau de l'espèce, de la structure du corps humain. D'autre part, la restauration de chaque partie anatomique était réglée par une propriété inhérente à celle-ci, qui en fixait les limites naturelles, y compris dans l'éventualité d'une pathologie.

La lecture de Pietro d'Abano va nous permettre de suivre concrètement les étapes principales de la transformation et de l'assimilation de l'aliment. Deux principes généraux président à la représentation. Le semblable attire le semblable : c'est en vertu de ce principe que les os, par exemple, de nature mélancolique vont attirer dans le «change» ce qui provient d'aliments dont les qualités sont celles de l'élément homologue de la mélancolie, à savoir la terre. Les qualités «semblables »

\footnotetext{
${ }^{9}$ On trouvera une synthèse sur le sujet de l'humide radical dans : C. Crisciani, «Aspetti del dibattito sull'umido radicale nella cultura del tardo medioevo (secoli XIII-XV) ", Actes de la "II Trobada internacional d'estudis sobre Arnau de Vilanova», éd. J. Perarnau, Barcelone, 2005, p. 333-380.

${ }^{10}$ Voir en particulier: J. Ziegler, «Ut dicunt medici: Medical Knowledge and Theological Debates in the Second Half of the Thirteenth Century ", Bulletin for the History of Medicine, 73, 1999, p. 208-237 ; Id., " Medicine and Immortality in Terrestrial Paradise », Religion and Medicine in the Middle Ages, éd. P. Biller-J. Ziegler, York, 2001, p. 201-214.
} 
prises en compte ne sont pas seulement le chaud, le froid, l'humide et le sec: s'y ajoutent le doux, l'amer, le visqueux, le piquant, l'astringent, etc., ce qui ouvre la voie à une diététique sophistiquée. L'attirance du semblable par le semblable n'exclut pas le jeu ordinaire des qualités entre elles, tel que la physique aristotélicienne le définit, à savoir que mises en contact dans un mélange, des qualités contraires interagissent pour donner au résultat final ses qualités propres. Toutefois, dans les processus de digestion, un autre principe fondamental intervient: l'action de la chaleur naturelle, d'une intensité variable selon les organes que détermine leur finalité spécifique, et réglée au niveau général par la faculté de nutrition, elle-même transmise par «l'esprit naturel» qui circule dans les veines. Les différentes étapes de la digestion correspondent ainsi à des degrés de cuisson et à des mécanismes sélectifs d'attraction et de répulsion.

Après ces préambules, nous pouvons suivre, avec Pietro d'Abano, le parcours de l'aliment. La mastication aide à sa transformation en le réduisant en petites parcelles (minima). Selon Aristote, dans Parties des animaux (II, 65a), il ne s'opère rien d'autre dans la bouche. Selon Avicenne, un début de digestion s'y produit sous l'effet de la chaleur. Notons que pour aucun de ces auteurs la salive ne joue un rôle dans la digestion. Assimilée à une humidité seconde, du type de la «rosée », issue en priorité de l'humeur phlegmatique, la salive humecte la langue pour faciliter l'élocution et aider à la perception des saveurs. C'est par ce seul dernier rôle qu'elle contribue à la fonction de nutrition. Son surplus est maintenu en attente dans les glandes salivaires. Grâce à l'acte de déglutition -à propos duquel les auteurs se demandent s'il relève d'un mouvement volontaire ou non-, les parcelles d'aliment issues de la mastication passent dans l'œsophage, puis dans l'estomac, où sous l'effet de la chaleur et de l'action mécanique d'éléments anatomiques appropriés, elles sont transformées en chyle. L'ouverture du pylore intervient alors, faisant descendre ce premier produit de la digestion dans les intestins, dont les multiples circonvolutions sont destinées à assurer la lente expulsion de ce qui doit l'être. Ce qui n'est pas expulsé, car conforme aux besoins du corps, passe dans les vaisseaux dits «mésentériques » de la paroi abdominale. La partie de chyle ainsi retenue y subit une nouvelle digestion, qui élabore un liquide rougeâtre, appelé chyme ${ }^{11}$. De vaisseaux en vaisseaux, le chyme passe dans la veine porte qui le mène au foie. Les veines issues de la veine porte y rentrent en contact avec les veines issues de la veine cave inférieure, et grâce à la chaleur propre au foie l'élaboration des humeurs se produit, à l'issue d'une cuisson. Comme le dit Avicenne, il faut imaginer un bouillon dans lequel il y a de l'écume, un dépôt, des choses brûlées, des choses bien cuites, des choses peu cuites. L'écume, c'est la bile; la chose peu cuite, le phlegme; la chose bien cuite, le sang; le dépôt, la mélancolie. Telles sont les humeurs dans leur état naturel. Quant aux humeurs brûlées (ou «adustes»), elles appartiennent au domaine du pathologique. Le degré de cuisson de chaque humeur n'est pas lié à une différence de durée dans son élaboration, car tout se produit simultanément : il est déterminé par la nature des aliments qui persiste dans le chyle, puis dans le chyme. La quantité et la qualité des humeurs dépendent donc directement de la nourriture. Les

\footnotetext{
${ }^{11}$ Le latin chimus vient du grec chumós, qui veut dire « suc », mais servait aussi en médecine à désigner les humeurs. Dans le vocabulaire médiéval, chimus désigne ce stade précis de la transformation du chyle dans les veines attenantes aux intestins.
} 
humeurs ainsi élaborées dans le foie forment la masse sanguine qui s'écoule dans les veines et, au terme d'une nouvelle purification qui a lieu dans le cœur, dans les artères.

À son entrée dans le système veineux, grâce à sa pénétration dans la veine cave inférieure, la masse sanguine se libère d'une substance aqueuse qui a aidé à cette pénétration : c'est l'urine, qui va suivre son propre cheminement à travers les reins et la vessie. Chez les femmes, c'est aussi à l'issue de cette seconde digestion effectuée dans le foie qu'est élaboré le sang menstruel, une sorte de sang imparfait qui sert toutefois de nourriture à l'embryon dans l'utérus maternel et contribue à la génération. D'autres types de prélèvements étaient intervenus au moment du passage des humeurs dans le système veineux. Une partie de la bile s'était dirigée vers la vésicule biliaire, à travers une veine issue de la veine porte, pour être utilisée en cas de besoin dans le nettoyage des intestins. Une partie de la mélancolie avait de même été recueillie dans la rate pour aider au resserrement du cardia et induire ainsi la sensation de faim. Du phlegme s'était répandu dans différents endroits du corps pour les lubrifier, en particulier dans le cerveau, d'où son surplus est expulsé par les fosses nasales.

La masse humorale qui constitue le sang subit une troisième digestion dans les veines et dans les vaisseaux jusqu'aux extrémités invisibles (ou pores) des plus petits d'entre eux, qui pénètrent dans les parties homogènes du corps. Après son élaboration dans les vaisseaux, sort de ces pores l'humidité seconde du premier type ou "sans nom», apte à se transformer si nécessaire en "rosée», enfin en «change», comme il a été dit précédemment. Au stade ultime, ou quatrième digestion, a lieu l'assimilation par les parties homogènes du corps de ce «change», qui, devenu semblable à elles, les régénère. C'est à ce stade ultime qu'un surplus, non nécessaire à cette régénération, est expulsé, chez les hommes, vers les testicules, où il se transforme en sperme. Qu'il s'agisse donc du sang menstruel, issu de la deuxième digestion ou du sperme, issu de la quatrième, les fluides indispensables à l'engendrement sont eux aussi étroitement dépendants de l'alimentation.

\section{Diététique et plaisir gustatif}

Tel que j'ai tenté de le reconstruire, ce modèle livré par Avicenne et Pietro d'Abano, structure, à quelques variantes de détail près, la représentation qu'ont les médecins médiévaux de la régénération du corps humain par la nourriture. Nul doute que pour eux chacun est ce qu'il mange. Le nombre des régimes qu'ils écrivent à partir du $\mathrm{XIII}^{\mathrm{e}}$ siècle témoigne suffisamment de l'importance accordée à la diététique. Mais n'est-ce pas cette diététique qui a semé la zizanie entre les deux sœurs siamoises qu'étaient à l'origine la médecine et la cuisine $?^{12}$ Sans doute pour ne pas passer que pour des rabat-joie et plaire à leur clientèle fortunée et indocile, les médecins choisirent de faire quelque concession au plaisir gustatif. Leur science leur en donnait les

\footnotetext{
${ }^{12}$ Sur le développement médiéval de la diététique, on se reportera aux travaux de Marilyn Nicoud, notamment: Conserver la santé au Moyen Âge, Les traités de diététique en Italie et en France (XIII ${ }^{e}-X V^{e}$ siècles), École française de Rome (BEFAR), sous presse. Sur les liens somme toute distendus entre médecine et cuisine, voir B. Laurioux, Une histoire culinaire $d u$ Moyen Âge, Paris, 2005, «Introduction », p. 32-34.
} 
moyens. Le médecin du $\mathrm{XV}^{\mathrm{e}}$ siècle, Jacques Despars, profite de l'ambiguïté du statut accordé à la déglutition pour introduire la notion de plaisir ${ }^{13}$. Avicenne dit, en effet, que l'acte de déglutir s'opère grâce à une attraction de la nourriture par les fibres de l'œsophage, qui relève à la fois d'un processus naturel et de la volonté. Selon Jacques Despars, la zone de partage passe par le goût, qui décide entre l'agréable et le désagréable, de manière diverse d'un individu à un autre : certains préfèrent le salé, d'autres le sucré ou l'acide. Tout dépend aussi du moment de la journée. Lorsqu'une nourriture est perçue comme agréable, elle est déglutie sans intervention de la volonté, par une attraction toute naturelle. Au contraire, la déglutition d'une substance désagréable est un acte volontaire, nécessitant l'arbitrage de la raison. Dans les cas extrêmes, il peut y avoir refus et vomissement. La gourmandise est ainsi médicalement justifiée, à condition qu'elle ne devienne pas une gloutonnerie sans mesure.

Comme tous les agréments de la vie, le plaisir gustatif a des vertus plus fondamentales. Le corps humain ne se limite pas à sa fonction digestive régénératrice. L'un des souffles qui l'anime, l'esprit vital élaboré par le cœur, est l'acteur des effets physiques des passions ou émotions, en particulier de la joie et de la tristesse. Ainsi que l'exprime le médecin parisien Jean Le Lièvre, au début du $\mathrm{XV}^{\mathrm{e}}$ siècle, les douceurs, tels qu'électuaires, dragées, poudres destinées à parfumer vins et hypocras, n'agissent pas sur le corps par la nature de leurs ingrédients, mais par leur saveur ${ }^{14}$. Le plaisir qu'elles procurent se répercute sur l'esprit vital, qui à son tour fortifie le cœur et stimule les actions apéritives et digestives. Il ne tenait donc qu'au médecin de faire converger son art du mélange avec celui du cuisinier. Encore convenait-il qu'il ne fît pas la confusion entre la diététique et une morale répressive de tous les plaisirs, selon une habitude qui perdure parfois aujourd'hui.

Danielle Jacquart

École Pratique des Hautes Études, section des Sciences historiques et philologiques

\footnotetext{
${ }^{13}$ Comm. Canon d'Avicenne, Lyon 1498, livre I, fen 1, doctrine 6, dernier chapitre (De operationibus).

${ }^{14}$ D. Jacquart, «Les douceurs du médecin Jean Le Lièvre (m. 1418)», "Docente natura », Mélanges de médecine ancienne et médiévale offerts à Guy Sabbah, éd. A. Debru-N. Palmieri, Saint-Étienne, 2001, p. 177-191.
} 